\title{
Cardiac Cachexia: Pathophysiology, Life Expectancy, Nutrition and Treatment
}

\section{Soni NR*}

Department of Quality Assurance (QA), Gujarat Technological University (GTU), India

*Corresponding author: Nirav R Soni, Department of Quality Assurance (QA), Gujarat Technological University (GTU), A-one Pharmacy College, Ahmedabad, India, Tel: +91 9033002567; E-mail: nirav_sonic@yahoo.com

Received date: December 06, 2017; Accepted date: December 12, 2017; Published date: December 15, 2017

Citation: Soni NR (2017) Cardiac Cachexia: Pathophysiology, Life Expectancy, Nutrition and Treatment. Cardiovasc Pharm Open Access 6: e138. doi: $10.4172 / 2329-6607.1000 \mathrm{e} 138$

Copyright: (c) 2017 Soni NR, This is an open-access article distributed under the terms of the Creative Commons Attribution License, which permits unrestricted use, distribution, and reproduction in any medium, provided the original author and source are credited.

\section{Editorial}

This comprehensive review offers fundamental knowledge of cardiac cachexia. It reveals the known detail of cardiac Cachexia, i.e. body muscle wasting, has long been documented as a serious complication of chronic sickness even with a very good appetite and high-calorie intake, some people lose muscle mass. Congestive cardiac failure (CCF) is a complex, dynamic disorder of many organ systems, involving the vascular, myocardial, neuro-hormonal, immune, gastrointestinal (GI), renal, CNS and musculoskeletal systems. It is the deterioration of this interactive, multisystem, network complex that results in the systemic inflammation and progressive wasting and atrophy of muscle and multiple other organ tissues, which is the trait of cardiac cachexia. The episode of wasting in CCF has been known for many centuries, but it hasn't been investigated extensively until recently. It is a common complication of CCF which is associated with poor prognosis, independently of functional disease severity, age, measures of exercise capacity, and left ventricular ejection fraction and may be cardiomyopathy. Cachectic CCF patients are weaker and fatigue earlier and suffer from generalized beating of bone, fat and lean tissue. This is due to both reduced skeletal muscle mass and spasm of skeletal muscle quality and regarding the pathophysiology of cardiac cachexia, there is escalating proof that immune abnormalities and neurohormonal may play a crucial role. Possible contributing factors contains malabsorption, the dietary deficiency, metabolic dysfunction, loss of nutrients via urinary and/or gastrointestinal tract (GIT), and metabolic imbalance leads to reduce energy. This passive congestion, in turn, would cause interstitial edema, and ascites, enlargent of liver which would then lead to reduced gastric capacity with feelings of abdominal fullness, early satiety, nausea and annorexia.

Cachectic CCF patients have augmented plasma levels of noradrenaline, adrenaline, and stress hormone like cortisol, and they show high plasma protein renin activity and increased plasma aldosterone levels. There are various number of studies have also shown that cardiac cachexia is linked to increased plasma levels of inflammatory cytokines (CK), such as tumor necrosis factor alpha (TNF- $\alpha$ ).

The available proof suggests this is a multifactorial neuro-endocrine and metabolic disorder or disturbances with a poor prognosis leads to be responsible for the development of the wasting process.
In the modern lifestyle some dietary factors that are antiinflammatory (i.e. reduce C-reactive protein) should be suggested: for example, fish oil supplements, walnut or olive, fruits and vegetables, flaxseed oil, garlic, turmeric (curcumin) and ginger, sunflower seeds, eggs, herring, nuts or zinc tablets, pineapple or bromelain supplements, grape juice or red wine in small amounts, antioxidant supplements such as ascorbic acid and $\alpha$-tochopherol, S-adenosyl-methionine, $\alpha$ lipoic acid, coenzyme Q10 (COQ10), mild to moderate exercise, abdominal fat lipolysis, stress reduction and regular teeth flossing. Green tea may also be helpful due to cause powerful natural antioxidants. Foods and supplements that are rich in omega-3 fatty acids docosahexaenoic acid (DHA) and eicosapentaenoic acid (EPA) tend to have a synergistic effect with drugs used for treating the major risk factors for CCF including hypertension and hyperlipidemia, as well as coronary artery diseases (CAD), stroke, and The older person's caregivers need to be grateful for the importance of 1) a high calorie, high protein diet plus supplements and 2) physical and mental activity as important parts of the patient's general treatment plan for cardiac cachexia and to the maintenance of social involvement and psychological well-being. Micronutrient and vitamin supplements are also helpful, especially in patients with malabsorption secondary to CCF. Lastly, the cornerstone in the prevention of cardiac cachexia remains the early diagnosis and prompt treatment of heart disease (HD) and the prevention of its progression to severe CCF.

In persons with CCF, activation of the neuroendocrine factors (NEF) such as proinflammatory cytokines such as TNF and other cytokines and catecholamines can further stimulate the metabolic rate of the tissue leads to the burning more calories. The destruction phase associated with stimulated resting energy spending would then predispose some CCF patients and it leads to developing cachexia. Working to find ways to break this sequence is one of the research goals of a number of researchers. The findings will in the future facilitate caregivers and practitioners to better manage this condition in aged persons. 